\title{
Forced oscillation techniques for measuring mechanical properties of the respiratory system
}

\author{
N B Pride
}

Department of Medicine, Royal Postgraduate Medical School,

Hammersmith Hospital, London W12 ONN

N B Pride

Correspondence to: Dr N B Pride

(reprints will not be available)
Airway function is usually assessed by tests of forced expiration or, in more specialised laboratories, by measuring airways resistance by whole body plethysmography. Plethysmography depends on the patients' cooperation and requires expensive and bulky apparatus. An alternative non-invasive technique for measuring airflow resistance, using forced oscillation, was introduced by DuBois and colleagues in the $1950 \mathrm{~s}^{1}$-almost at the same time as body plethysmography-but did not become as widely established in clinical physiology. The biomechanical principles of forced oscillation are generally less familiar than those underlying conventional measurements of airways resistance and lung compliance, and the technical requirements for obtaining accurate pressure and flow signals and the subsequent signal processing are more demanding. Recent advances in pressure transducer and microcomputer technology, however, have removed many of the earlier problems with signal collection and analysis. Because most oscillation methods require less cooperation from the subject and less bulky apparatus than body plethysmography the balance of advantage between the two methods has altered and we can expect the oscillation technique to become more widely used in clinical physiology.

\section{Methods of applying forced oscillation}

Forced oscillation techniques deduce the mechanical properties of the respiratory system from the response to small externally produced oscillatory forces. From the responsemeasured as the instantaneous pressure-flow relationship (impedance)-flow resistance and the reactance (the combined effect of elastance and inertance) of the respiratory system can be computed. Because these mechanical properties of the lungs are non-linear it is important that only small external forces are applied.

Most commonly, forced oscillation is applied at the airway opening by a loudspeaker and the pressure-flow relation is also measured at the airway opening ("input" impedance-see fig 1). Alternative techniques apply an oscillatory signal to the surface of the chest wall and measure the induced flow response at the airway opening or they oscillate at the airway opening and measure flow at the chest wall ("transfer" impedance). ${ }^{2}$ Transfer impedance has certain technical advantages but requires the subject to be seated in a head out plethysmograph and will not be further discussed.

The simplest form of forced oscillation technique applies a single sinusoidal signal during breath holding (fig 2). The impedance
Figure 1 Schematic representation of typical apparatus and analysis used for measuring input impedance. Pmo-mouth pressure; $\dot{V}$ mo-flow at mouth; MTLV-midtidal lung volume. The loudspeaker may be driven to generate a sinusoidal oscillation at one frequency, a sequential series of sinusoidal oscillations at different single frequencies, or, as in most modern applications, random noise, which with Fourier transform analysis allows simultaneous analysis of the imposed pressure-flow relations over a wide range of

frequencies.

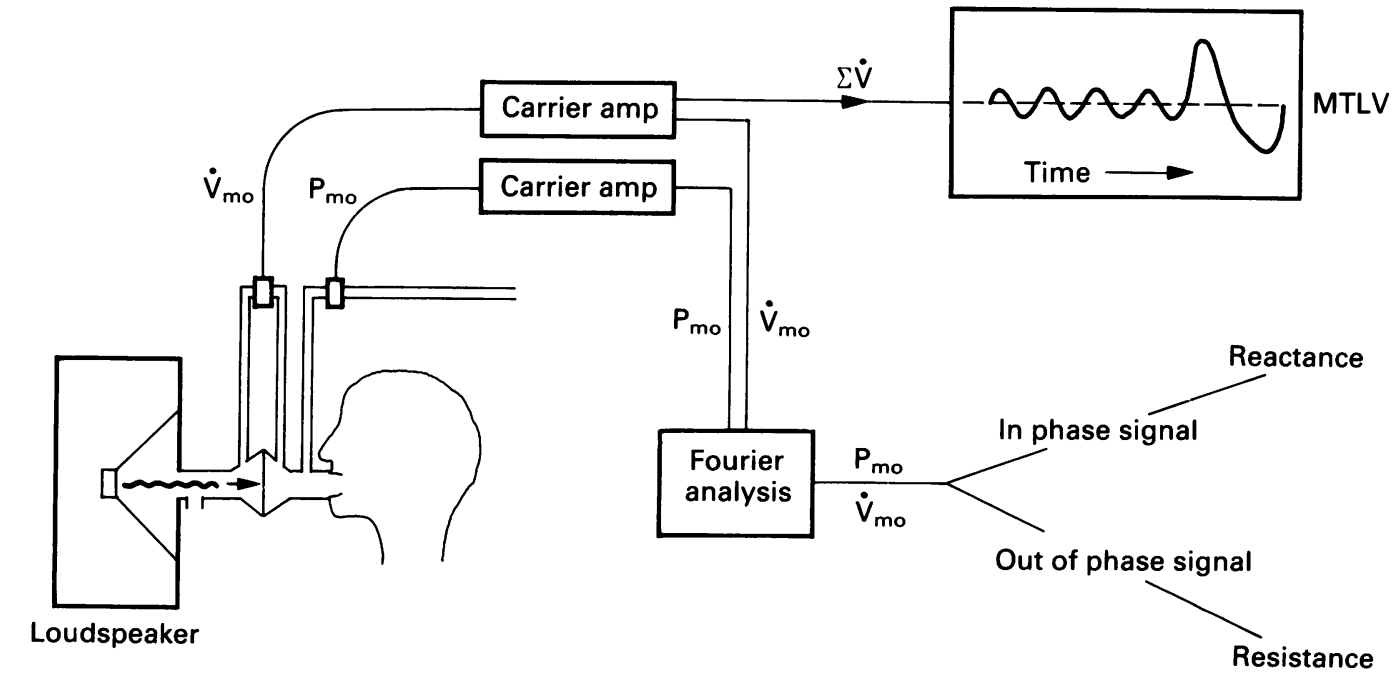


Figure 2 Change in mouth flow $(\dot{V})$ and pressure $(P)$ during monosinusoidal oscillation with the subject holding his breath. In this example peaks and troughs of $\dot{V}$ and $P$ signals are in phase, but $\dot{V}$ may lead or lag the $P$ signal. In most modern systems a much more complex signal is generated and analysed, forced random noise being superimposed on the flows of tidal breathing.

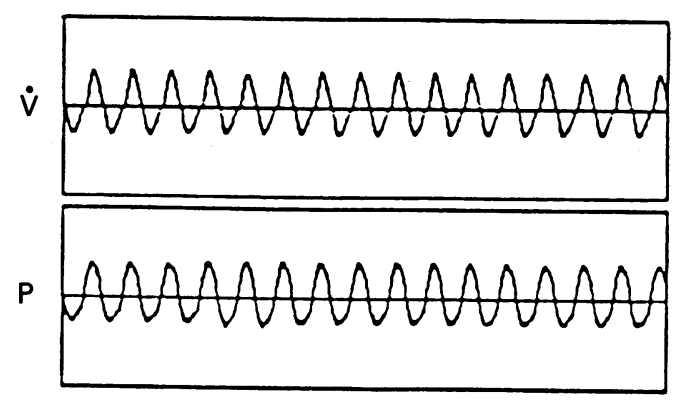

of the subject's respiratory system at this frequency is obtained by dividing the instantaneous value of pressure by the flow at the mouth. The mechanical properties of the normal respiratory system vary with the frequency of oscillation and this characteristic is amplified by respiratory disease. This frequency dependence can easily be examined by the oscillation method. Initially the change in impedance with frequency was obtained by repeating the measurement during application of a sequential series of sinusoidal frequencies. Most modern systems, however, obtain this information much more rapidly by applying forced random noise (or programmed pseudorandom noise) generated by a loudspeaker during spontaneous tidal breathing. This requires a much more sophisticated microcomputer analysis, which uses fast Fourier transform analysis to decompose the full range of induced pressure and flow signals contained in the random noise into their individual frequency components followed by ensemble averaging. ${ }^{3}$ In this application the pressureflow response to forced oscillation at the airway opening (a peak to peak pressure signal of $1-2 \mathrm{~cm} \mathrm{H}_{2} \mathrm{O}$ ) has to be distinguished from the pressure developed as a result of the resistance of the apparatus and the flows of tidal breathing. The ability to distinguish the response to external forcing depends on the difference between the frequency of the applied oscillation and that of the subject's own tidal breathing and the period over which measurements are made. With short periods of data collection (typically 16 seconds) the lowest frequency at which impedance can be measured in normal subjects at rest is $4 \mathrm{~Hz}$; this frequency is higher if there is pulmonary disease or increased ventilation and in children and infants.

\section{Analysis of the pressure and flow response} to forced oscillation

Regardless of the precise method of applying forced oscillation, the primary measurements are the pressure and flow produced at the airway opening at a given frequency by the external applicd forces. The instantaneous pressureflow relationship (impedance of the respiratory system, Zrs) developed is a consequence of the total mechanical properties of the respiratory system; it is further analysed into its in phase and out of phase components at each applied frequency. ${ }^{2}$

The in phase component of input impedance (sometimes called the "real" part) is due to the flow resistive properties of the total respiratory system (Rrs, the sum of extrathoracic airway, intrathoracic airway, lung tissue, and chest wall resistance, all arranged in series). The derived value for Rrs is usually a time average of inspiratory and expiratory resistance, including changes in glottal diameter over the several breaths of a 16 second period of measurement. Although Rrs provides a less specific measurement of subglottal airway calibre than body plethysmography, in practice absolute changes in Rrs after bronchoactive drugs are similar. A potential weakness of the oscillation technique is that there is no automatic accompanying measurement of absolute lung volume. The mouth flow signal, however, can easily be integrated to provide continuous information on minute ventilation (fig 1). After measurement of Rrs inspiratory capacity can be measured. If total lung capacity is measured separately, values for the functional residual capacity (FRC) and, more relevantly, midtidal lung volume (MTLV) at which Rrs was measured can then be derived and used to calculate specific resistance $($ SRrs $=$ Rrs.MTLV). This measurement of absolute lung volume-or at least of inspiratory capacity-is particularly important when acute changes in MTLV are induced by bronchoconstrictor or bronchodilator drugs or by changes in posture.

The out of phase component of impedance is less familiar. It is usually called reactance (Xrs) but sometimes the "imaginary" part of impedance. It can be negative (pressure peak lagging flow peak) or positive (pressure peak leading flow peak), depending on the frequency of oscillation (fig 3 ). In terms of the conven-
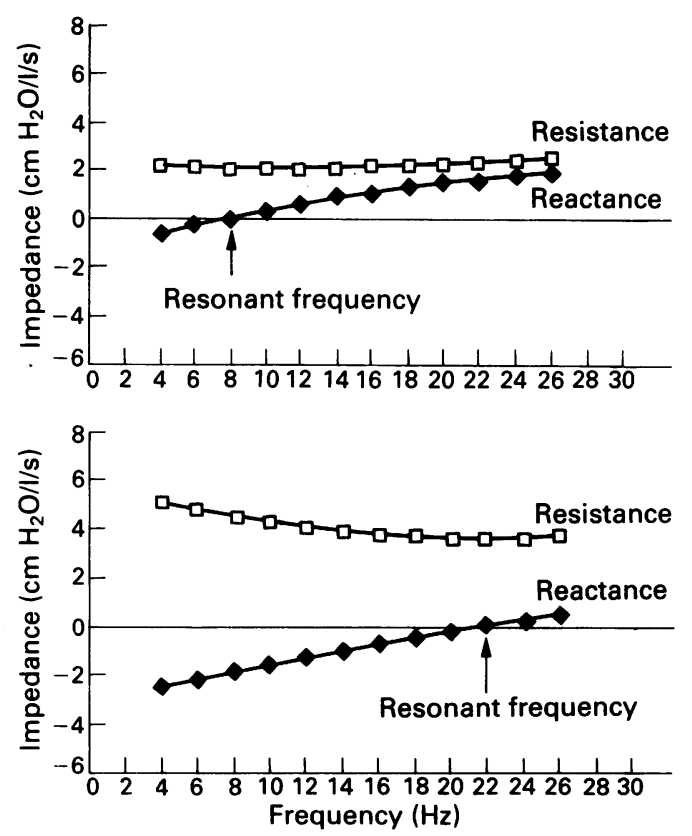

Figure 3 Typical changes in total respiratory resistance and reactance at different applied oscillation frequencies in a normal subject (top panel) and a patient with chronic obstructive lung disease (bottom panel). In a patient with airways obstruction resistance is higher at all frequencies than in a normal subject and decreases with increasing frequency; reactance is lower at all frequencies and resonant frequency (frequency at which reactance $=0$ ) is increased. 
tional three compartment electrical analogue model of the mechanics of the respiratory system, reactance is a combined function of elastance (pressure required to change volume) and inertance (pressure required to accelerate flow) of the respiratory system. At low frequencies elastance is dominant (and leads to the lagging of pressure behind flow); as frequency is increased inertance becomes increasingly dominant (and pressure then leads flow). Consequently reactance at low frequencies is negative and increases as frequency increases. When reactance is zero the pressure and flow responses are perfectly in phase; this is the resonant frequency of the respiratory system. Unfortunately with input impedance reactance cannot be subdivided into its compliance and inertance components when Rrs is frequency dependent.

\section{Applications of the technique \\ The main current application in clinical physiology of the forced oscillation technique is as a simple method for obtaining the flow resistive properties of the respiratory system at different frequencies during tidal breathing. Its simplicity by comparison with plethysmo- graphy or oesophageal pressure measurement is attractive. More extensive data are usually analysed with oscillation than with either of these two techniques-or indeed with the air- way interrupter technique.}

\section{AIRWAYS OBSTRUCTION}

In contrast to the relative constancy of Rrs at different frequencies in healthy subjects, in patients with intrapulmonary airway obstruction $\mathrm{Rrs}$ is increased at the lower applied frequencies but falls with increasing applied frequencies (fig 3). The findings are similar in asthma and in chronic obstructive lung disease. Values of reactance (Xrs) are lower than in healthy subjects and become positive only at higher frequencies, so that resonant frequency is increased (fig 3). In the presence of inhomogeneity of mechanical properties of the lungs, resistance falls with increasing frequency, so this finding in intrapulmonary obstruction was expected. The usual input impedance technique, however, exaggerates the frequency dependence of Rrs. Thus Rrs falls with increasing frequency of oscillation in patients with upper airway obstruction without any suspicion of intrapulmonary disease. ${ }^{4}$ This occurs because there is increased dissipation of the applied oscillatory signal in the upper airway (chiefly the cheeks and floor of mouth) whenever resistance is increased further down the airway. The pressure-flow properties of the subject's upper airway, which themselves are frequency dependent, then play a greater part in determining the total respiratory impedance. The contribution of the cheeks and mouth to total impedance can be reduced, but not eliminated, by firm support of the cheeks and the floor of the mouth with the palms and fingers.

A recent innovation has been to develop a small head plethysmograph. ${ }^{5}$ In this applica- tion the oscillation signal is generated in a 30 35 litre plethysmograph (head box) and the subject breathes from the air in the headbox via a pneumotachograph and mouthpiece. Thus oscillations are applied both to the external surface of the cheeks and neck and, via the mouthpiece and pneumotachograph, to the internal surface of the oropharyngeal airway, reducing oscillation induced variations in the transmural pressure of the upper airway. This technique should allow the overall importance of upper airway impedance in influencing frequency dependent behaviour to be assessed. Until this is done the change of Rrs with frequency cannot be interpreted in terms of the response of central or peripheral intrapulmonary airways as was originally hoped. ${ }^{6}$

\section{RESTRICTIVE DISEASE OF THE LUNGS AND CHEST} WALL

Some studies have been made in patients with fibrosing alveolitis ${ }^{7}$ and with kyphoscoliosis and ankylosing spondylitis. ${ }^{8}$ These have shown modest increases in Rrs and reduced values of reactance with an increase in resonant frequency. Surprisingly, distinctive patterns of mechanical abnormality in diseases conventionally regarded as having very different mechanical origins have not been found with the simple input impedance technique. This may be because upper airway impedance becomes important whenever increased pressures are required to ventilate the lungs.

\section{OTHER APPLICATIONS}

The apparatus can be adapted for measurements of input impedance in different postures and with different gas mixtures and can be applied to various airway openings (mouth, nose, tracheostomy, endotracheal tube). Obvious areas of application include acute respiratory illness, respiratory disease in children, anaesthesia, and intensive care. So far there is very little information on these applications. The method can also be applied to epidemiological surveys because little training of the subjects is required. Measurement of the total resistance to breathing is probably the correct measurement to use in external loading studies. Preliminary work has suggested that it may be useful for evaluating nasal airflow resistance.

In the research laboratory there is the hope that a more elaborate analysis of transfer impedance, carried out up to very high applied frequencies, could distinguish the contributions of airway and tissue properties to the total impedance without interference from upper airway impedance. ${ }^{2}$

The full potential of oscillation techniques in clinical respiratory physiology remains to be exploited.

1 DuBois AB, Brody AW, Lewis DH, Burgess DF. Oscillation mechanics of lungs and chest in man. J Appl Physiol 1956; 8:587-94.

2 Peslin R. Methods for measuring total respiratory impedance by forced oscillations. Bull Eur Physiopathol Respir 1986;22:621-31. 
3 Landser FJ, Nagels J, Demedts M, Billiet L, Van de Woestijne KP. A new method to determine the frequency characteristics of the respiratory system. J Appl Physiol 1976;41:101-6.

4 Van Noord JA, Wellens W, Clarysse I, Cauberghs $M$, Van de Woestijne KP, Demedts $M$. Total respiratory resistance and reactance in patients with upper airway obstruction. Chest 1987;92:475-80.

5 Peslin R, Duvivier C, Didelon J, Gallina C. Respiratory impedance measured with head generator to minimize upper airway shunt. J Appl Physiol: Respirat Environ Exercise Physiol 1985;59:492-501.
6 Pimmel RL, Tsai MJ, Winter DC, Bromberg PA. Estimating central and peripheral respiratory resistance. $J A p p l$ Physiol 1978;45:375-80.

7 Van Noord JA, Clement J, Cauberghs M, Mertens I, Van de Woestijne KP, Demedts M. Total respiratory resistance and reactance in patients with diffuse interstitial lung disease. Eur Respir J 1989;2:846-52

8 Van Noord JA, Cauberghs $M$, Van de Woestijne KP, Demedts $M$. Total respiratory resistance and reactance in ankylosing spondylitis and kyphoscoliosis. Eur Respir J 1991;4:945-51.

\section{Practice makes perfect}

There were more than 1000 radiologists in the audience. The great pathologist Averill Liebow was preparing to project radiographs on the vast screen and we, the cognoscenti of the chest radiograph, were there, on show, to make the expected brilliant diagnoses, thus to stimulate and inspire the radiological hoi polloi in the auditorium. My fellow "expert" at this illustrious gathering was Ben Felson, surely the finest chest radiologist in the world at that time. I was pleased and honoured to be sharing the limelight with these two transatlantic "giants" and I'm ashamed to admit that my ego did wallow a little in the penumbra of their fame as the show began in an atmosphere crackling with anticipation. Ben took the first case with his accustomed panache-a scar carcinoma at the right apex simulating tuberculous infiltration to perfection. The master was not fooled for a moment and Liebow's histological material confirmed the confident diagnosis. We were off to a good start! The microphone was now placed in front of me but Ben Felson grabbed it to tell one of his stunningly dirty jokes. The audience was helpless with laughter, and even Averill Liebow smiled as he projected the next radiograph. My turn. Bolstered by my Brompton background and a fatally swollen ego I felt entirely confident and at ease. A posteroanterior and lateral chest radiograph of a 28 year old white woman with no clinical symptoms. A chance finding. The lesion was in the posterior segment of the left lower lobe, $2 \mathrm{~cm}$ in length, ovoid, homogeneous and solitary. I went through the well worn drill. A film taken six months previously was normal, thus ruling out sequestration, congenital cyst, and many other long standing abnormalities. I asked about joint disease and the sinuses. Had she lived in hydatid country or dwelt among the fungal flora of the Mid West? I drew a blank. Feeling a little less comfortable by now I touched on the atypical primary, the solitary metastasis, and various interlobar rarities and other unlikely possibilities and finished up toying miserably with an isolated deposit of amyloid, the refuge of the intellectually barren. I wanted to go home. Averill's histological slide showed the characteristic features of a rheumatoid nodule (the patient apparently developed joint symptoms six months later). I was not enjoying myself.
Back at the Brompton a week later I was talking to a colleague in the radiology department when George Simon came along the corridor with his idiosyncratic scurrying gait, clutching the inevitable armful of radiographs. "How was Miami?" - "Oh fine, fine, but I had a rather tricky case at the conference quiz ..." I had only just started to recount the salient features of my case but George was already moving off and showing little sign of interest in my words. As he reached the doorway he said over his shoulder "Oh, that would be a rheumatoid nodule ..." and vanished through the door. I was so stunned that it took me a minute or two to recover and chase after him, but he'd gone. My God! He'd diagnosed my case from the other side of the Atlantic without even hearing about its radiographic features!

I didn't catch up with George Simon again for several days, but I finally ran him to earth ferreting about in the radiography museum. "George, how the hell did you know that was a rheumatoid nodule?" He went on sorting films while saying in his flat sonorous monotone, "When I first saw that film in San Diego I got it wrong and I got it wrong again in Los Angeles, but when they showed it to me in Boston I did get the right diagnosis!" Oh well, practice makes perfect, I suppose, though I didn't say that to George, who wasn't the flippant sort.

In the States the radiological quiz has been refined and perfected over the years and is a deservedly popular attraction at conferences, as indeed it is at our conferences. Nowadays the "experts" work hard in the run up to their performances, avidly reading the latest books and journals in an effort not to be caught out in front of a hopeful and very updated audience. Everyone gains in knowledge from these public forays, especially the platform experts, whose enforced scouring of contemporary publications brings them right up to date on many a topic they might otherwise pass by, and they arrive at the table brimming with knowledge. They cannot any longer rely on "practice" to bring perfection. The practice itself must be perfect. At most major conferences that usually is the situation and I have great admiration for the erudite participants and their academic enterprise. 